Case Report

\title{
Neurological and Hematological Recovery Following Copper Replacement in Severe Copper Deficiency-induced Myelopathy and Cytopenia
}

\author{
Christoph Roland Seifert ", Ieva Didrihsone, Christian Isensee \\ Department of Neurology, Hermann-Josef-Hospital, Academic Educational Hospital of the University of Aachen, Erkelenz, Germany
}

Email address:

cseifert@hjk-erkelenz.de (C. R. Seifert)

${ }^{*}$ Corresponding author

\section{To cite this article:}

Christoph Roland Seifert, Ieva Didrihsone, Christian Isensee. Neurological and Hematological Recovery Following Copper Replacement in Severe Copper Deficiency-induced Myelopathy and Cytopenia. Clinical Neurology and Neuroscience. Vol. 5, No. 1, 2021, pp. 1-4. doi: $10.11648 /$ j.cnn.20210501.11

Received: December 21, 2020; Accepted: December 29, 2020; Published: January 18, 2021

\begin{abstract}
Background: Copper deficiency can lead to a severe myelopathy and cytopenia. The myelopathy can lead to inability to walk by paresis and deafferentation. The anemia can as well be severe with the need for blood transfusions. Beside innate reasons it occurs years after surgeries of the intestine and following disturbed resorption. It can also occur due to excessive intake of zinc that binds copper. An early diagnosis following copper substitution can lead to restitution. It can clinically be mistaken for a vitamin B12 deficiency. This circumstance can lead to a delayed treatment. Case: A 67 years old woman with severe gait disturbances, sensory ataxia was admitted to our hospital. She had the diagnosis of vitamin B12 deficiency. B12 was elevated and metabolites were in normal range. The electrophysiology showed a deafferentation of the legs. Objective: This case report provides detailed information about the course of diagnosis and the treatment of a severe copper deficiency with symptomatic myelopathy and cytopenia in an elderly woman. Methods: We performed a literature research with keywords "spinal cord disease, gait disturbances, elevated lactate in cerebrospinal fluid, anemia". We did blood tests and CSF on a regular basis combined with clinical assessments and electrophysiology. Results: Serum copper was not detectable. CSF copper was reduced $(8.7 \mu \mathrm{g} / \mathrm{l}$, Ref.: $14.2-109 \mu \mathrm{g} / \mathrm{l})$, CSF lactate was elevated $(4.69 \mathrm{mmol} / \mathrm{l}$, Ref.: $1.1-2.4 \mathrm{mmol} / \mathrm{l})$. The patient had a cytopenia (Hb 6.6 g/dl, Ref.: 12-16 g/dl, MCV 130 fl, Ref.: 80-96 fl, leucocytes $2.310^{3}$, Ref.: 4.3-10 103/ 1 l). Vitamin B12 amounted to $891 \mathrm{pg} / \mathrm{ml}$ (Ref.: 191-663 pg/ml). The somatosensory evoked potential (SEP) study showed absent cortical responses after stimulation of the sural nerves bilaterally. Due to the diagnosis of a myelopathy and bone marrow dysfunction due to copper deficiency we substituted copper-histidine subcutaneously. This led to a marked improvement of symptoms, an increase of serum copper levels $(141 \mu \mathrm{g} / \mathrm{l})$, CSF copper levels $(14.1 \mu \mathrm{g} / \mathrm{l})$, hemoglobin $(11.9 \mathrm{~g} / \mathrm{dl})$, leukocyte count $\left(11.410^{3} / \mu \mathrm{l}\right)$ and a normalization of CSF lactate. Conclusion: Elevated CSF lactate might be an indicator for treatable myelopathy due to copper deficiency in patients with afferent gait disturbances. A timely substitution can lead to a marked improvement of even severe symptoms.
\end{abstract}

Keywords: Copper Deficiency, Anemia, Spinal Cord Disease, Gait Disturbances Elevated CSF Lactate, Hypocupremia

\section{Introduction}

The combination of acquired spinal cord dysfunction and cytopenia of blood cells is a syndrome caused by paraneoplastic and lymphoproliferative syndromes, HIV, vitamin B12 and folic acid deficiencies or copper deficiency
[1]. Copper is available in a wide variety of animal and vegetable food, so that inadequate levels (hypocupremia) occur either through disturbed resorption, excess of zinc that binds copper or due to a damaged or removed intestine [2]. Neurological symptoms encompass severe sensory ataxia caused by lesions of dorsal columns with decreased vibratory and joint position senses as well as lesions of the lateral 
columns with spastic paraparesis. A peripheral neuropathy with paresthesias and hypoesthesia has also been described [3]. The pathological features of hypocupremia are driven by the impairment of the multiple vital enzymatic processes in which copper acts as a cofactor, for example in the respiratory chain in mitochondria, the glucose metabolism, protection from oxidative stress, connective tissue biogenesis and neurotransmitter synthesis [4].

Copper deficiency associated myelopathy was first described only 20 years ago [5], and has not yet been included in the routine diagnostic workup of myelopathies accompanied by cytopenia. As a consequence, a median time of 12 months was found from onset of symptoms to diagnosis [6]. However, timely detection of hypocupremia is paramount to the prevention and improvement of neurological deficits. We present a case with hypocupremia induced myelopathy and cytopenia following a gastric resection, rapidly improving after substitution of copper.

\section{Case Report}

In December 2019, a 67-year old woman presented with significant rapidly progressing difficulty in walking. Her past medical history included partial gastrectomy due to a stomach ulcer (1988) with multiple revision surgeries (gastrojejunostomy, hemicolectomy) and lastly subtotal residual gastrectomy in 2017. Few months later in 2017 she noticed a slight tingling in her feet. Physical examination at the time of admission in 2019 revealed symmetrical hypaesthesia and thermhypaesthesia in the lower legs. While ankle reflexes were missing, knee jerks as well as arm deep tendon reflexes were brisk bilaterally. Pyramidal signs were negative. Nerve conduction studies were normal. The somatosensory evoked potential (SEP) study showed absent cortical responses after stimulation of the sural nerves bilaterally and the right posterior tibial nerve. On stimulation of the left posterior tibial nerve, a small cortical response could be recorded with a delayed latency. The MRI of the cervical region was without certain pathology. Laboratory examination revealed macrocytic anemia and leukopenia (9.7 $\mathrm{g} / \mathrm{dl}$ und $2.410^{3} / \mu \mathrm{l}$ ). Serum vitamin B12 concentration was normal due to a recently started substitution. Holotranscobolamin or methylmalonic acid were not further examined. Vitamin B12 deficiency was suggested. Accordingly a substitution of vitamin B12 was initiated. A lumbal puncture was not performed.

In April 2020 the patient presented again with rapidly progressing symptoms. She was no longer able to stand or even walk. The neurological examination revealed a severe sensory ataxia of the legs, arms were asymptomatic. Severe hypoesthesia with thermhypesthesia and zero vibration sense was found in the lower extremities, and epicritic sensation was significantly impaired with diminished joint position sense down from the iliac crests. Muscle reflexes were brisk in the lower extremities (again with missing ankle jerks) and slightly decreased in upper extremities. There was no reduction of muscular force on detailed testing, and no pyramidal signs.
Cortical SEPs on sural and posterior tibial nerve stimulation were diminished and delayed. Laboratory examination revealed macrocytic anemia and leukopenia with severe neutropenia (table 1). Accordingly, the patient received an erythrocyte concentrate. She reported improvement of fatigue with no effect on mobility.

Further diagnostic included evaluation of paraneoplastic and lymphoprolipherative syndromes as well as vitamins and copper. Total vitamin B12, holotranscobolamin, folic acid as well as methylmalonic acid and homocysteine were all within a normal range. Bone marrow biopsy showed an increased number of immature myeloid and erythroid cells, suggestive of a nutrient deficiency. Antineuronal and paraneoplastic antibodies in the cerebrospinal fluid were negative. The lactate level was increased in the cerebrospinal fluid (4.69 mmol/1, Ref.: 1.1-2.4 mmol/1). Copper was not detectable in serum, decreased in urine ( $8 \mu \mathrm{g} / 24 \mathrm{~h}$, Ref.: $10-60 \mu \mathrm{g} / 24 \mathrm{~h})$ and in the CSF (8.7 $\mu \mathrm{g} / 1$, Ref.: 14.2-109 $\mu \mathrm{g} / \mathrm{l})$ [7]. Caeruloplasmin in the serum was not detectable (table 1). Zinc was found within the normal range $(1.16 \mu \mathrm{g} / \mathrm{ml}$, Ref.: 0.6-1.2 $\mu \mathrm{g} / \mathrm{ml})$.

For logistic reasons, we started copper substitution by oral substitution with copper gluconate. It resulted in slow improvement of cytopenia ( $\mathrm{Hb}$ 6.6-10.4 g/dl, leukozytes 2.3-5.5 $\left.10^{3} / \mu \mathrm{l}\right)$ and slow increase of the copper serum levels $(<$ $4-26 \mu \mathrm{g} / \mathrm{dl}$ ) within 9 days. Therefore, a substitution with a subcutaneous copper-histidine (6 syringes of $2.4 \mathrm{mg}$ copper-histidine, from Alte Apotheke Stuttgarter Strasse 51, 70469 Stuttgart, Germany was performed. This drug was prepared after the formula from the pharmacy of the university hospital in Heidelberg [8]. As a result, serum and urine copper levels increased as well as in the CSF, while CSF lactate decreased as a sign of the normalization of the glucose metabolism (table 2). Subsequently leukocytes, particularly neutrophils, returned to normal and erythrocytes and hemoglobin increased along with an increase of reticulocytes (table 1). Neurological examination showed a marked improvement of the mobility. The patient was able to walk short distances without aids and long distances with a rollator. Both hypoesthesia and thermaesthesia improved. She also reported a slight vibratory sensation at the iliac crest bilaterally. She felt more secure walking and was discharged with oral $5 \mathrm{mg}$ copper gluconate daily.

On follow-up 5 months later, she was able to walk without any walking aids, showing only slight residual gait instability. For longer outdoor distances she admitted using a rollator. She had gained weight from 40 to $44 \mathrm{~kg}$. Clinical examination revealed vibration sensation $2 / 8$ at the medial malleoli on both sides and $3 / 8$ and $4 / 8$ at the knees right and left. She still reported also a hypaesthesia and a thermhypaesthesia in her right leg and felt burning allodynia in the feets on touch. Deep tendon reflexes were brisk and symmetric in upper and lower extremities. Laboratory examination revealed a slight normocytic anemia (Hb $11.9 \mathrm{~g} / \mathrm{dl}$, MCV $94.2 \mathrm{fl}$ ) with normal neutrophil counts (table 1). Coeruloplasmin in the serum and urine was normal, and the free copper increased. CSF copper was $14.1 \mu \mathrm{g} / \mathrm{l}$ and lactate was within a normal range $(1.97$ $\mathrm{mmol} / \mathrm{l})$ (table 2). The renal panel showed increased urea (76 
$\mathrm{mg} / \mathrm{dl}$, Ref.: 0-71) and creatinine (1.8 mg/dl, Ref.: 0.7-1.2).

Table 1. Hematological restitution due to copper substitution.

\begin{tabular}{|c|c|c|c|c|c|c|}
\hline & $\begin{array}{l}\text { Before } \\
\text { transfusion }\end{array}$ & $\begin{array}{l}\text { Before } \\
\text { Copper p.o. }\end{array}$ & $\begin{array}{l}\text { Before } \\
\text { Copper i.m. }\end{array}$ & $\begin{array}{l}\text { At the time } \\
\text { of discharge }\end{array}$ & $\begin{array}{l}\text { External } \\
\text { follow-up }\end{array}$ & $\begin{array}{l}\text { In-house } \\
\text { follow-up }\end{array}$ \\
\hline & $14 / 04 / 2020$ & $20 / 04 / 2020$ & $29 / 04 / 2020$ & 05/05/2020 & $04 / 06 / 2020$ & $05 / 10 / 2020$ \\
\hline Red blood cell count (Ref.: 4-5.2 mil/ $/ \mu \mathrm{l})$ & 1.7 & 2.6 & 2.9 & 2.9 & 3.6 & 4.0 \\
\hline $\mathrm{Hb}$ (Ref.: $12-16 \mathrm{~g} / \mathrm{dl})$ & 6.6 & 9.4 & 10.4 & 10.0 & 11.4 & 11.9 \\
\hline MCH (Ref.: 28-33 pg) & 38.8 & 35.6 & 36.0 & 35.0 & 31.1 & 30.1 \\
\hline MCV (Ref.: 80-96 fl) & 130.0 & 109.8 & 115.9 & 114.0 & 103.3 & 94.2 \\
\hline Reticulozytes (Ref.: 5-15\%o) & & 30.0 & & 65.0 & 27.0 & 16.0 \\
\hline Leukocytes (Ref.: 4.3-10 103/ $/$ l) & 2.3 & 3.9 & 5.5 & 7.7 & 10.6 & 11.4 \\
\hline Neutrophils (Ref.: $1.5-810^{3} / \mu 1$, severe neutropenia $<0.510^{3} / \mu 1$ ) & 0.4 & 1.6 & & 3.5 & 7.1 & 5.8 \\
\hline
\end{tabular}

$\mathrm{Hb}=$ hemoglobin, $\mathrm{MCH}=$ mean corpuscular hemoglobin, $\mathrm{MCV}=$ mean corpuscular volume, p.o.=per osl, i.m.=intramuscular, color gradient from dark gray to white representing grading pathological to normal values

Table 2. Copper levels in serum and lactate in the CSF due to copper substitution.

\begin{tabular}{lllll}
\hline & Before copper p.o. & Before copper i.m. & At the time of discharge & In-house follow-up \\
\hline & $\mathbf{2 0 / 0 4 / 2 0 2 0}$ & $\mathbf{2 9 / 0 4 / 2 0 2 0}$ & $\mathbf{0 5 / 0 5 / 2 0 2 0}$ & $\mathbf{0 5 / 1 0 / 2 0 2 0}$ \\
\hline Serum copper (Ref.: $68-169 \mu \mathrm{g} / \mathrm{dl}$ ) & $<4$ & 26 & 72 & 141.0 \\
Serum free copper (Ref.: $<40 \mu \mathrm{g} / \mathrm{dl}$ ) & Not detectable & 5.2 & 26.7 & 41.6 \\
Ceruloplasmin (Ref.: $16-45 \mathrm{mg} / \mathrm{dl})$ & $<3$ & 6.6 & 14.7 & 28.4 \\
Urine copper (Ref.: $10-60 \mu \mathrm{g} / 24 \mathrm{~h}$ ) & 8 & & 55 & 34.0 \\
CSF copper (Ref.: $14.2-109 \mu \mathrm{g} / \mathrm{l})$ & 8.7 & & 10.1 & 14.1 \\
Lactate in the CSF (Ref.: $1.1-2.4 \mathrm{mmol} / \mathrm{l})$ & 4.96 & 3.15 & 1.97 \\
\hline
\end{tabular}

$\mathrm{CSF}=$ cerebrospinal fluid, p.o.=per os, i.m.=intramuscular, color gradient from dark gray to white representing pathological to normal values

Because of the normalized copper but impaired renal function in the serum a reduction of copper gluconate substitution to $2 \mathrm{mg}$ daily was prescribed. Follow-up visits were arranged every three months showing stable improvement and normal serum copper levels as well as normal renal and liver function tests.

\section{Discussion}

Copper is an essential micronutrient involved in hematopoiesis, myelin sheet formation and collagen and elastin synthesis [2,9]. Copper accumulates in small intestine and kidneys, while the brain and other tissues have unusually low levels resulting in progressive neurodegeneration and connective tissue anomalies [10]. Acquired hypocupremia is associated with myelopathy and cytopenia as seen also in our case. Whether insufficiencies of anastomoses requiring multiple revision operations were due to disturbed copper metabolism in our patient via ineffective collagen synthesis remains to be discussed. There was no family history of possible Menkes disease in our patient and its X-linked recessive trait (strongly favoring manifestations in males) renders a genetic cause unlikely.

The acidic environment in the stomach facilitates freeing the copper bond to foodstuff [11]. After deliberation, copper is absorbed in the small intestine by passive and active transport. Excessive zinc intake leads to the upregulation of metallothionein, which in turn binds copper and retains it within the enterocyte blocking its further absorption into the plasma [1]. Thus, using e.g. zinc-containing denture adhesives may cause hypocupremia. The same principle is being used therapeutically in the treatment of Morbus Wilson [13].
Previous gastrectomy in our patient suggests impaired absorption as the cause of hypocupremia. Although our patient used zinc-containing denture adhesives, zinc in the serum was within a normal range. Copper however was significantly decreased in all probes.

Increased lactate levels in the cerebrospinal fluid are typically associated with mitochondrial disorders [14]. Copper is a cofactor of cytochrome $\mathrm{c}$ oxidase in the mitochondrial respiratory chain [15]. Copper deficiency induces a switch to anaerobic energy production routes and subsequently an increase of lactate [16]. To our knowledge our patient provides the first documented association between serum and CSF copper normalization combined with normalization of CSF lactate. The latter is routinely measured and may thereby serve as an indicator of hypocupremia and as a marker for the restoration of mitochondrial function in the CNS.

Furthermore, the substitution of copper leads to rapid restoration of the hematopoiesis and prevention of further worsening of neurological symptoms. The neurological improvement depends on timely substitution. Unfortunately by the time of diagnosis the damage of neural structures is often already partly irreversible so that the response of substitution is more subjective than objective and usually sensory [3]. Indeed our patient showed a more rapid recovery of the cytopenia. Encouragingly however also the neurological deficits improved radically, so that on the follow-up examination the patient ambulated with only slight instability without major walking aids in her daily life. From the perspective of patient, the greatest improvement occurred to her mobility and sensation. She once again felt her upper legs completely.

To our knowledge this is the first reported case of a 
successful therapy of copper deficiency-induced myelopathy and cytopenia in an elderly patient. Furthermore it provides a detailed documentation of the clinical course and laboratory values upon the substitution of copper levels. Evidently, a rapid diagnosis and swift substitution of copper lead to successful recovery of the patients also in advanced age. Including copper level measurement in the initial diagnostic work-up for spinal ataxia allows identification of a safely treatable and otherwise devastating disorder.

\section{Conclusion}

With respect to diagnostics we suggest that patients with afferent gait disturbances should be screened and potentially monitored for elevated CSF lactate that might indicate treatable copper deficiency and myelopathy. Moreover an incomplete restitution in cases of diagnosis of Vitamin B12-induced myelopathy or cytopenia notwithstanding sufficient substitution should lead to reevaluation with regard to copper deficiency. The recommendation for a wider screening derives from the circumstance that often anamneses are insufficient because of patients not remembering surgeries many years ago or lacking documentation. In our case the surgeries were reported by the patient, but the extend came to light weeks after the admission. Despite copper deficiency-induced myelopathy and cytopenia being rare conditions, probably underdiagnosed, a need to establish guidelines for the diagnostic course of gait disturbances including measuring copper levels evolves. Especially in not-neurological wards, for example in geriatrics without a neurological cooperation, gait disturbances are ubiquitous and diagnosis often fogged by multimorbidity. Therefore unsparing use of blood tests that include copper levels could prevent deficiencies before they occur. And even symptomatic copper deficiency-induced myelopathy and cytopenia can be treated successfully like this case report underlines.

\section{References}

[1] C. L. Rohm, S. Acree, and L. Lovett, "Progressive myeloneuropathy with symptomatic anaemia," BMJ Case Reports, vol. 12, no. 12, Dec. 2019, doi: 10.1136/bcr-2019-230025.

[2] N. and A. EFSA NDA Panel (EFSA Panel on Dietetic Products, "Scientific Opinion on Dietary Reference Values for copper," EFSA Journal, vol. 13, no. 10, pp. 4253-51 pp, Oct. 2015, doi: $10.2903 /$ j.efsa.2015.4253.

[3] N. Kumar, "Copper deficiency myelopathy (human swayback)," Mayo Clinic Proceedings, vol. 81, no. 10, pp. 1371-1384, 2006, doi: $10.4065 / 81.10 .1371$.
[4] L. v. Puchkova, M. Broggini, E. v. Polishchuk, E. Y. Ilyechova, and R. S. Polishchuk, "Silver Ions as a Tool for Understanding Different Aspects of Copper Metabolism," Nutrients, vol. 11, no. 6, Jun. 2019, doi: 10.3390/nu11061364.

[5] B. Schleper Hans Joerg Stuerenburg, B. Schleper, and H. J. Stuerenburg, "Copper deficiency-associated myelopathy in a 46-year-old woman," J Neurol, vol. 248, pp. 705-706, 2001.

[6] A. A. Gabreyes, H. N. Abbasi, K. P. Forbes, G. Mcquaker, A. Duncan, and I. Morrison, "Hypocupremia associated cytopenia and myelopathy: A national retrospective review," European Journal of Haematology, vol. 90, no. 1. pp. 1-9, Jan. 2013, doi: 10.1111/ejh.12020.

[7] X. Feng Wang and M. S. Cynader, "Pyruvate Released by Astrocytes Protects Neurons from Copper-Catalyzed Cysteine Neurotoxicity," 2001.

[8] T. Hoppe-Tichy, M. Lorke, T. Hoppe-Tichy, T. H. Nguyen, and B. W. Hentze, "Kupferhistidin-Lösung zur Behandlung von Menkes' Kinky Hair Syndrom: Herstellung und Stabilität," Pharmazie, vol. 60, pp. 205-207, 2005, [Online]. Available: https://www.researchgate.net/publication/233516212.

[9] A. W. Zimmerman, J.-M. Matthieu, R. H. Quarles, R. O. Brady, and J. M. Hsu, "Hypomyelination in Copper-Deficient Rats Prenatal and Postnatal Copper Replacement," Archives of Neurology, vol. 33, no. 2, pp. 111-119, 1976, [Online]. Available: http://archneur.jamanetwork.com/.

[10] Z. Tümer and L. B. Møller, "Menkes disease," European Journal of Human Genetics, vol. 18, no. 5, pp. 511-518, May 2010, doi: 10.1038/ejhg.2009.187.

[11] R. A. Wapnir, "Copper absorption and bioavailability," The American Journal of Clinical Nutrition, vol. 67, no. 5 Suppl, pp. 1054S-1060S, 1998.

[12] G. Ranucci, F. di Dato, M. I. Spagnuolo, P. Vajro, and R. Iorio, "Zinc monotherapy is effective in Wilson's disease patients with mild liver disease diagnosed in childhood: A retrospective study," Orphanet Journal of Rare Diseases, vol. 9, no. 1, Mar. 2014, doi: 10.1186/1750-1172-9-41.

[13] M. Magner et al., "Elevated CSF-lactate is a reliable marker of mitochondrial disorders in children even after brief seizures," European Journal of Paediatric Neurology, vol. 15, no. 2, pp. 101-108, Mar. 2011, doi: 10.1016/j.ejpn.2010.10.001.

[14] W. W. Wainio, C. vander Wende, and N. F. Shimp, "Copper in Cytochrome c Oxidase," THE JOURNAL OF BIOLOGICAL CHEMISTRY, vol. 234, no. 9, 1959, Accessed: Nov. 01, 2020. [Online].

[15] S. Nargund, J. Qiu, and C. T. Goudar, "Elucidating the role of copper in CHO cell energy metabolism using 13C metabolic flux analysis," Biotechnology Progress, vol. 31, no. 5, pp. 1179-1186, Sep. 2015, doi: 10.1002/btpr.2131. 